\title{
Forewords
}

\section{Network design and optimization}

\section{Building, optimizing and dimensioning networks of tomorrow}

The design of communication networks witnesses presently a revolution, the origin of which is to be found in the raise of the so-called Communication and Information Techniques (for french speaking users, the acronym TIC is becoming more and more in use), which make use of multimedia applications. All these keywords which are frequently used nowadays are a testimony for the advent of multiservices networks. The multiservices nature of the traffics has given rise to the notion of Quality of Service (QOS), which is yet another major keyword to understand the variety of studies around communication network.

Another source of the richness of these studies is the variety of communication techniques which have to interoperate. The classical ISDN uses a circuit based technique, while connectionless IP networks are deployed over fixe as well as mobile networks. Moreover, satellite networks appear which raise a lot of issues unknown in traditional technologies.

This is to say that the teletraffic engineer is faced with an increasing collection of new problems to deal with, for which new analysis techniques are to be proposed, giving rise to exciting challenges. The goal of this special issue of the Annals of Telecommunications it to provide the reader with a modest sample of such problems and techniques, and to show the kind of results which may be expected. The issue is more especially devoted to network planning and dimensioning, which deliberately excludes such important topics as real-time control, and more generally traffic management techniques. A complete special issue to come could be devoted to these points?

Even with such a restricted area, the papers presented here do not cover the huge diversity of possible topics. The partial survey proposed here to the reader reflects the choices of the guest editors - who are the only responsible for them.

Planning and dimensioning are two complementary aspects of the same topic. This is typical in each of the studies presented therein. For instance, the paper by A. FEIRRERA et al. (see below) proposes an algorithm to optimize routing in a satellite network. The results may be used as well to dimension the network (how much traffic can I offer with a certain QoS constraint, or what capacity to be provided).

The first step in the design of a network is to gather information about potential customers and about the traffics they generate. For the traditional telephone service, the operators make use of a large set of observations they have stored from the beginning of the service. Now, emerging services cannot directly benefit from this large base of knowledge, users behavior being by nature unknown. The same holds for all emerging technologies.

Related to this point, the paper by Catherine GLOAGUEN presents an original method, which allows a statistical description of the flows and of their geographic profile. The stochastic geometry gives a model which somehow resembles the statistical mechanics approach : a few parameters describe the traffic, taking account of the randomness of the individual connection demands and give of them a macroscopic description - just as the temperature, for instance, takes account of the energy and motion of individual molecules of a gas. This yields a prevision of the traffic flows between the switches of the network. Evidently, this kind of model does not intend to give precise answers to short term planning decisions, such as trunk sizing. Rather, they bring precious information to medium or long term planning engineers, about the impact of structural choices, and about strategic planning options.

As soon as the traffic matrices are obtained, the network may be designed, based upon optimality criteria. However, there remains an important issue which is too often ignored, at the network planning level, and which concerns routing procedures. As traffics are not known with a high precision, and as the network topology may vary, the routing protocol must adapt to the actual traffic pattern and topology. The first paper to address such issues is by Jérome GEFFARD and Marc Van OUDHEUSDEN. The paper addresses the basic routing problem: given a traffic matrix (i.e. a set of 
origin-destination flows to be carried) and a network topology with given link capacities, how to determine a set of paths which maximizes to quantity of traffic to be carried. The problem is known to be "complex" (i.e., according to the formalism, NP-hard). The authors propose heuristic solutions, the efficiency of which are discussed. It must be stressed that, although the authors quote the ATM technology as an example of the use of this approach, this one is really quite general, independent of the technology (ATM, Frame Relay, fixe or mobile).

The paper by Walid BEN AMEUR and Bernard LIAU illustrates another aspect of routing issues. Here, the question of the choice of a metric is addressed. A routing algorithm makes use of the shortest path between the origin and the destination, the shortest path being found according to some criterion : each link is allocated a "length", which can be any metric (the actual length, the load, a performance measure, or any other). The question they solve concerns the existence of metrics ensuring that the shortest paths make use of all links (thus guaranteeing the effective use of all the investments). They show that such a metric may be built, using only "small" integer measures - which happens to be an important requirement for the deployment in an IP-based network.

The third example concerns the very special case of satellite networks (paper by Afonso FEIRRERA et al.). The so-called Leo (low earth orbit) satellite network introduce another problem, which is that the network topology is continuously changing, due to satellite movements. When intersatellite links are possible, a connection is likely to be routed along a few different paths successively. The problem can be compared with the operation by which a mobile user is served (the handover operation). To ensure a given QoS, the availability of the next path must be guaranteed. The authors propose an algorithm for route reservation, the efficiency of which is asserted.

The last paper (by Sami TABBANE) is devoted to yet another technological field. In a mobile network, an important requirement is to quickly locate a moving customer - the roaming operation. This amount to build and maintain a huge database, using tracking or paging operations. The paper presents a survey of existing methods, emphasizing the pros and cons of each one. It then proposes adaptive methods, where the mobile terminal participates to the process by identifying its location, or where the network takes advantage of repetitive mobility patterns. The performance of the methods are compared via a simulation procedure.

As is should be evident from this short presentation, this special issue does not pretend to address the whole field of network planning and dimensioning. Nevertheless, the sample of papers collected here illustrates perfectly the great variety of the approaches and of the techniques which are used : discrete-event simulation, mathematical programming, both exact and heuristic, algorithmic methods, probability theory, etc.).

Gérard HÉBUTERNE

Institut National des Télécommunications 9, rue Charles Fourier, 91011 Evry (France)

Jean-Yves LEBoudeC

École Polytechnique Fédérale de Lausanne EPFL-1 CA, INN (Ecublens), Ch-1015 Lausanne (Suisse) 